Orthopäde $2017 \cdot 46: 610-616$

DOI 10.1007/s00132-017-3418-2

Online publiziert: 31. März 2017

(c) Der/die Autor(en) 2017. Dieser Artikel ist eine Open-Access-Publikation.

CrossMark

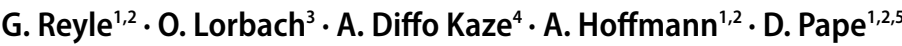

'Orthopädische Klinik des Centre Hospitalier de Luxembourg, Akademisches Lehrkrankenhaus der Universitätskliniken des Saarlandes, Luxembourg, Luxemburg

${ }^{2}$ Sports Medicine Research Laboratory, Luxembourg Institute of Health, Luxembourg, Centre Médical de la Fondation Norbert Metz, Luxembourg, Luxemburg

${ }^{3}$ Orthopädische Universitätsklinik des Saarlandes, Homburg/Saar, Deutschland

${ }^{4}$ Faculty of Science, Technology and Communication, University of Luxembourg, Luxembourg, Luxemburg

${ }^{5}$ Cartilage Net of the Greater Region, Homburg/Saar, Deutschland

\section{Verhinderung einer Fraktur der Gegenkortikalis bei aufklappender Osteotomie}

\section{Anteroposteriore Bohrung als Lösungsansatz}

\section{Die Entlastungsbohrung im Bie- gungsscharnier verzögert das Auftreten einer lateralen Kor- texfraktur bei der aufklappenden Tibiaosteotomie um etwa $2^{\circ}$, scheint jedoch bei der Korrektur einer Varus- fehlstellung durch die valgisierende tibiale Umstellungsosteotomie keine Bedeutung zu haben.}

Die aufklappende Osteotomie des Tibiakopfes (HTO) ist eine weit verbreitete Methode zur Behandlung einer medialen Gonarthrose [2-5]. Neben den allgemeinen Operationsrisiken der HTO kann die Fraktur der (lateralen) Gegenkortikalis eine Ursache für verzögerte Heilung, einen Korrekturverlust und ungewollte Slopeveränderungen sein [1, 6-12]. Die Inzidenz dieser Fraktur kann laut Literatur zwischen 0,3 und $35 \%$ schwanken $[7,13]$.

Takeuchi et al. [1] etablierte eine Klassifikation der Frakturarten im Biegungsscharnier der Gegenkortikalis („hinge“) und beschrieb in seiner Studie mit 104 HTO-Patienten eine Inzidenz von $25 \%$ radiologisch nachweisbaren Frakturen im Biegungsscharnier der lateralen Kortikalis.

Zur Minderung des Frakturrisikos haben Kessler et al. [7] bei der lateral zuklappenden HTO das Verfahren

\begin{tabular}{|c|c|c|c|c|c|c|}
\hline & $\begin{array}{l}\text { Gruppe } 1 \\
\text { Synbone } \\
\text { ohne } \\
\text { Bohrung }\end{array}$ & $\begin{array}{l}\text { Synbone } \\
\text { mit } \\
\text { Bohrung }\end{array}$ & $\begin{array}{l}\text { Sawbone } \\
\text { ohne } \\
\text { Bohrung }\end{array}$ & $\begin{array}{l}\text { Gruppe } 2 \\
\text { Sawbone } \\
\text { mit } \\
\text { Bohrung }\end{array}$ & $\begin{array}{l}\text { Kadaver } \\
\text { ohne } \\
\text { Bohrung }\end{array}$ & $\begin{array}{l}\text { Gruppe } 3 \\
\text { Kadaver } \\
\text { mit } \\
\text { Bohrung }\end{array}$ \\
\hline 1 & 3 & 5 & 3 & 3 & 3 & 2 \\
\hline 2 & 2 & 7 & 3 & 5 & 1 & 6 \\
\hline 3 & 3 & 8 & 1 & 6 & 3 & 2 \\
\hline 4 & 4 & 4 & 2 & 5 & 4 & 4 \\
\hline 5 & 3 & 5 & 2 & 6 & 3 & 3 \\
\hline 6 & 4 & 8 & 1 & 4 & 2 & 6 \\
\hline 7 & 2 & 4 & 2 & 5 & 3 & 5 \\
\hline 8 & 3 & 4 & 3 & 3 & 7 & 7 \\
\hline 9 & 2 & 6 & 2 & 5 & 2 & 5 \\
\hline 10 & 4 & 4 & 3 & 4 & 1 & 2 \\
\hline$\varnothing$ & 3 & 5,5 & 2,2 & 4,6 & 2,9 & 4,2 \\
\hline
\end{tabular}

Abb. 1 ॥ Zusammenfassung der maximalen Aufklappwinkel bis zur Fraktur der Gegenkortikalis für alle Gruppen in Grad $\left({ }^{\circ}\right)$ und Durchschnittswerten (Ø) 

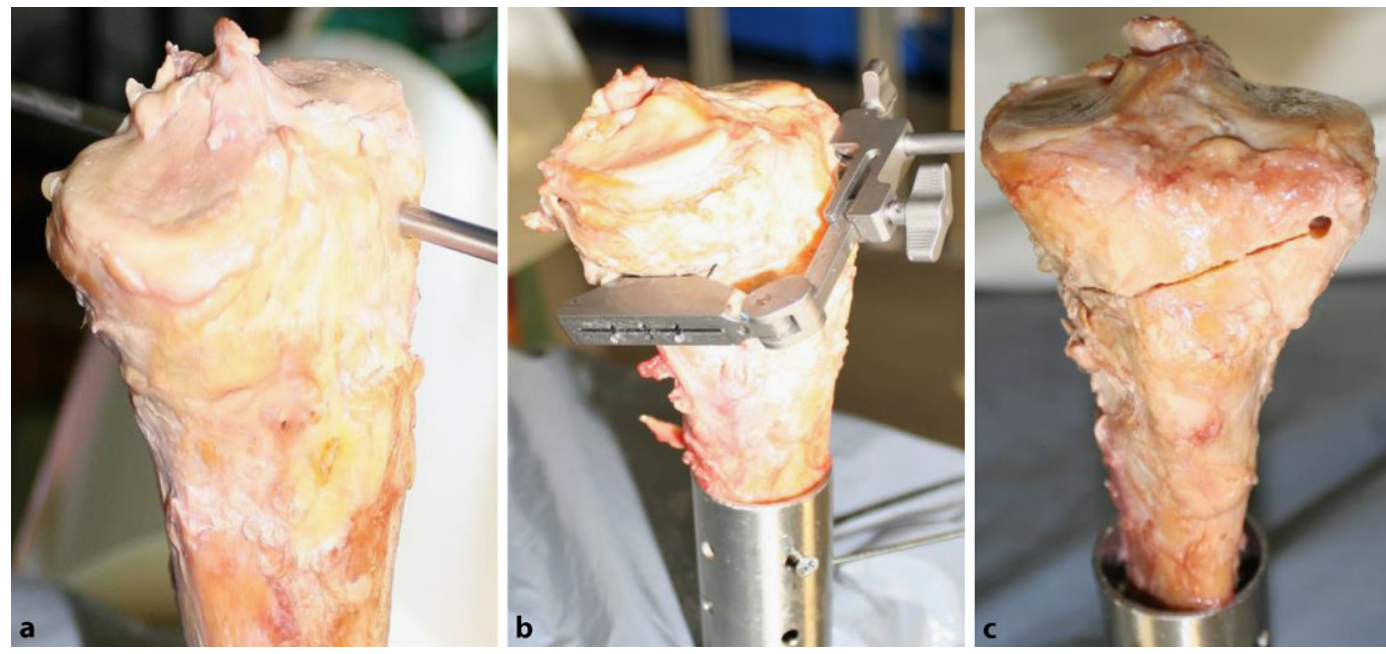

Abb. $2 \triangleleft$ Hinge-drillTechnik mit dem Arthrex Hing-Pin-Set ${ }^{\circledR}$. a Setzen des Bohrers in anteroposteriore Richtung durch das laterale Biegungsscharnier des Tibiakopfs (Hinge $\mathrm{Pin}^{\circledR}$ ). b Montage der Sägelehre. c Ergebnis nach Sägen und Entfernung von Bohrer und Sägelehre

der Entlastungsbohrung im Biegungsscharnier evaluiert und gezeigt, dass hierdurch eine Steigerung des Korrekturwinkels um ca. $50 \%$ möglich war, ohne das Frakturrisiko der (medialen) Gegenkortikalis zu erhöhen.

Aus klinischen und biomechanischen Studien ist bekannt, dass beim Aufspreizen des Osteotomiespalts einer HTO die Gegenkortikalis als elastisches Biegungsscharnier dient. Bei größeren Korrekturwinkeln wird die Kapazität des Knochens zur elastischen Deformierung regelmäßig überschritten, so dass die Deformierung der Gegenkortikalis plastisch wird und Frakturen der Gegenkortikalis auftreten, die zum Korrekturverlust der Osteotomie führen können [8, 14-16]. Eine anteroposteriore Entlastungsbohrung („hinge drill“) am Ende der horizontalen Osteotomie soll die Kapazität des Biegungsscharniers zur elastischen Deformierung erhöhen und im Idealfall Frakturen der Gegenkortikalis vermeiden [7].

Die vorliegende Studie untersucht den Effekt dieser Bohrung auf die Integrität der Gegenkortikalis in Abhängigkeit eines sich schrittweise vergrößernden Korrekturwinkels während der medial aufklappenden Osteotomie des Tibiakopfes.

\section{Material und Methoden}

\section{Prüfkörper}

Die beiden Osteotomieverfahren wurden an drei unterschiedlichen Prüfkörpern durchgeführt (• Abb. 1):
Die erste Gruppe (Gruppe 1, $n=20$ ) bestand aus Synbone ${ }^{\circledR}$-Knochen (SYNBONE AG, Karlihof, CH-7208 Malans). Sie sind aus Polyurethan gefertigt, weisen eine dem menschlichen Knochen ähnliche Struktur mit Kortikalis und Spongiosa auf und sind hauptsächlich für die Erlernung von Osteosynthese- und Osteotomieverfahren konzipiert. Sie weisen eine geringere Spongiosa- und Kortikalisdichte als Sawbone-Modelle auf und erwiesen sich in den Versuchen als sehr elastisch. Daten zu den biomechanischen Eigenschaften dieser Modelle existieren laut Hersteller nicht.

In der zweiten Gruppe (Gruppe 2, $n=20$ ) wurden Sawbone-Knochenmodelle der 4. Generation verwendet (Sawbones, Pacific Reasearch Laboratories, Inc., Vashon, WA). Diese Modelle weisen ähnliche mechanische Eigenschaften wie menschlicher Knochen auf und eliminieren den Einflussfaktor der interindividuellen Schwankungsbreite der Knochendichte im Vergleich zu humanen Präparaten [17-19].

Die dritte Gruppe (Gruppe 3, $n=20$ ) bestand aus Kadaverknochen des anatomischen Instituts der Universitätsklinik Homburg/Saar (12 Frauen und 8 Männer). Die Spender der Präparate wiesen ein durchschnittliches Alter von 73 Jahren auf. Die nichtfixierten Tibiapräparate wurden unter Erhalt des Periosts vom Weichteilmantel befreit (-Abb. 2a-c).

\section{Osteotomietechnik}

Die 3 Gruppen wurden je zur Hälfte in der Standardtechnik und zur anderen Hälfte mit Hilfe des Hing-Pin ${ }^{\circledR}$-Systems von Arthrex ${ }^{\circledR}$ osteotomiert.

Die Tibiae aller Kunstknochen (Gruppe 1-2) wurden einer biplanaren HTO in Anlehnung an die Empfehlungen der „knee expert group“ (KNEG) der Arbeitsgemeinschaft für Osteosynthesefragen $(\mathrm{AO})$ für die aufklappende valgisierende Tibiakopfosteotomie unterzogen $[3,5,20]$. Die horizontale Osteotomie endete 5-8 mm entfernt von der intakten Gegenkortikalis und $2 \mathrm{~cm}$ unterhalb des lateralen Tibiaplateaus. Bei den Kadaverknochen wurde zugunsten einer besseren Reproduzierbarkeit der Osteotomien und zur Minimierung von Einflussvariablen lediglich die uniplanare horizontale Osteotomie in Anlehnung an die Technik von Fowler et al. durchgeführt [21].

\section{Präparation des Biegungs- scharniers (",hinge drill")}

Für die Präparation des Biegungsscharniers wurden die Bohrschablone, der Hinge-Pin-Bohrer (5 mm Durchmesser) und das kalibrierte Sägezielgerät der Firma Arthrex ${ }^{\circledR}$ aus dem iBalance ${ }^{\circledR}$-HTOSet verwendet. Da die Osteotomien allesamt ohne Weichteilmantel durchgeführt werden konnten, war eine präund intraoperative Bildwandlerkontrolle nicht nötig. Die Präparationsschritte entsprachen den Empfehlungen der Ope- 
Orthopäde 2017 · 46:610-616 DOI 10.1007/s00132-017-3418-2

(c) Der/die Autor(en) 2017. Dieser Artikel ist eine Open-Access-Publikation.

\section{G. Reyle · O. Lorbach · A. Diffo Kaze · A. Hoffmann · D. Pape}

\section{Verhinderung einer Fraktur der Gegenkortikalis bei aufklappender Osteotomie. Anteroposteriore Bohrung als Lösungsansatz}

\section{Zusammenfassung}

Hintergrund. Bei einer Osteotomie mit größeren Korrekturwinkeln wird die Kapazität des Knochens zur elastischen Deformierung regelmäßig überschritten, so dass die Deformierung der Gegenkortikalis plastisch wird und Frakturen der Gegenkortikalis auftreten, die zum Korrekturverlust der Osteotomie führen können. Eine anteroposteriore Entlastungsbohrung („,hinge drill“) am Ende der horizontalen Osteotomie soll die Kapazität des Biegungsscharniers zur elastischen Deformierung erhöhen und im Idealfall Frakturen der Gegenkortikalis vermeiden. Material und Methoden. Je 20 Synbone-, Sawbone- und Tibiakadaverknochen wurden einer aufklappenden Osteotomie des
Tibiakopfes in Standardtechnik unterzogen. Bei je 10 Tibiae pro Gruppe wurde zusätzlich eine Entlastungsbohrung am lateralen Ende der Osteotomie durchgeführt. Auftretende Frakturen der Gegenkortikalis wurden photographisch und radiologisch dokumentiert. Die Frakturen wurden gemäß der TakeuchiKlassifikation den Typen 1-3 zugeteilt. Ergebnisse. Bei allen 3 Untersuchungsgruppen war der Korrekturwinkel beim Auftreten der Fraktur der Gegenkortikalis im knöchernen Biegungsscharnier durch die zusätzliche Entlastungsbohrung größer. Der durchschnittliche Korrekturwinkel aller Tibiae ohne Entlastungsbohrung lag bei 2,7 und konnte mit Entlastungsbohrung um 2,1 $(+77,8 \%)$ auf $4,8^{\circ}$ vergrößert werden. Schlussfolgerung. Bei kleineren Korrekturwinkeln zur Entlastung knorpelsanierender Eingriffe bei gerader Beinachse kann die Entlastungsbohrung am knöchernen Biegungsscharnier der Osteotomie eine Fraktur verhindern. Bei Achskorrekturen zur Behandlung von Varusgonarthrosen scheint dieses Verfahren keinen frakturverhindernden Effekt aufzuweisen.

\section{Schlüsselwörter}

Tibiakopf $\cdot$ Entlastungsbohrung $\cdot$ Hohe aufklappende Tibiaosteotomie $\cdot$ Laterale Kortexfraktur · Biegungsscharnier

\section{Prevention of lateral cortex fractures in open wedge high tibial osteotomies. The anteroposterior drill hole approach}

Abstract

Background. In osteotomies with larger correction angles, the capacity for elastic deformation is frequently exceeded, resulting in plastic deformation and fracture of the opposite cortex, which may lead to subsequent loss of correction. An anteroposterior drill hole at the apex of the horizontal osteotomy (= hinge) is supposed to increase the capacity of the bony hinge for elastic deformation and ideally to prevent fractures of the opposite cortex.

Materials and methods. A high tibial osteotomy (HTO) using standard surgical technique was performed in 20 each of Synbones, Sawbones, and human cadaver tibial specimens. In 10 specimens per group, an additional anteroposterior hinge drilling was performed at the apex of the horizontal osteotomy. All fractures of the opposite cortex were photographically and radiographically documented. All fractures were classified according to fracture types $1-3$ of the Takeuchi classification.

Results. Regardless of the study group, all tibial bones with an additional hinge drilling achieved larger correction angles during the spreading of the wedge until a fracture of the opposite cortex occurred. The average correction angle of all specimens without the drill hole was $2.7^{\circ}$, which increased to $4.8^{\circ}$ with the hinge drill (increase by $77.8 \%$ ). In correction angles exceeding $5^{\circ}$, all specimen showed a hinge fracture regardless of the presence or absence of a hinge drill.

Conclusions. The hinge-protecting effect is restricted to small correction angles, i. e., to unload cartilage repair regions in the absence of severe malalignment. For the treatment of varus gonarthrosis, there is no fractureprotecting effect from a hinge drill.

\section{Keywords}

Tibial head · Stress-relief hole $\cdot$ High tibial osteotomy · Lateral cortex fracture · Hinge drill rationsanleitung des HTO Hinge-PinSystems der Firma Arthrex ${ }^{\circledR}$.

Zunächst wurde der laterale Endpunkt der Osteotomie mittels einer Schablone in Abhängigkeit vom Querdurchmesser des Tibiaplateaus festgelegt. Dieser Endpunkt lag bei allen Präparaten auf Höhe des proximalen Tibiofibulargelenks, etwa $2 \mathrm{~cm}$ unterhalb des lateralen Tibiaplateaus und ca. $8 \mathrm{~mm}$ von der lateralen Kortikalis entfernt. Ein 2,4-mmFührungsdraht wurde sagittal parallel zum Tibiaplateau eingebracht und mit einem 5-mm-Hohlbohrer überbohrt, so dass eine wenige Millimeter dicke Gegenkortikalis erhalten bleiben konnte (- Abb. 2c). In diesen Bohrkanal wurde der Hinge Pin platziert, der durch seine medialseitige Abflachung als Sägestopp diente. Nun wurde das Zielgerät für den horizontalen Schnitt an den Pin montiert. Das Zielgerät wurde analog zur Standardtechnik etwa $3,5 \mathrm{~cm}$ unterhalb des medialen Plateaus angesetzt und mit 2 Führungsdrähten temporär fixiert. Das Sägeblatt der Osteotomie stieß so am Endpunkt der Osteotomie lateral auf den Hinge Pin, die Integrität der Gegenkortikalis war gewährleistet.

\section{Aufspreizen der Osteotomie}

Es erfolgte das Aufspreizen aller Präparate mit einer Reihe von Flachmeißeln schrittweise um jeweils $1 \mathrm{~mm}$ an der Keilbasis ( Abb. 3a, b). Für einen gegebenen Korrekturwinkel $(\mu)$ ist die Höhe (h) der Keilbasis von der Tibiaplateaubreite (TPB) in Höhe der horizontalen Osteotomie abhängig. Die Höhe kann berechnet werden, indem die TPB mit der Tangente 


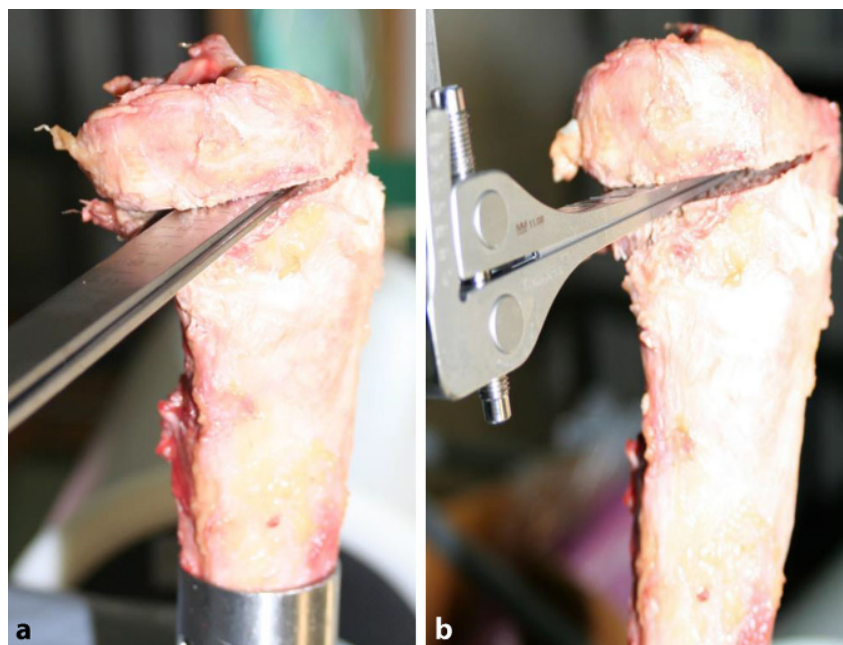

Abb. 3 A Osteotomie und Mobilisierung des Osteotomiespalts. a Schrittweises Aufspreizen mit Hilfe der Flachmeißel, $\mathbf{b}$ ab $3^{\circ}$ mit dem kalibrierten Osteotomiespreizer

(tan) des Korrekturwinkels multipliziert wird: $h=\mathrm{TPB} \times \tan \mu$. Bei einer durchschnittlichen TPB von $75 \mathrm{~mm}$ gilt folgende Faustregel: Pro Millimeter Keilbasishöhe ändert sich der Korrekturwinkel um $0,8-1^{\circ}[22]$.

Zur Messung des Grades der Aufspreizung wurde ab $3^{\circ}$ Aufspreizung zusätzlich ein kalibrierter Osteotomiespreizer mit Anzeige der Gradzahl verwendet (- Abb. 3b).

Dabei den Kunstknochen eine Fraktur der Kortikalis mit dem bloßen Auge sichtbar war, wurde diese fotografiert und der Frakturverlauf mit entsprechender Gradzahl der Aufspreizung zum Zeitpunkt des Auftretens der Fraktur dokumentiert. Bei den Kadaverpräparaten war wegen des intakten Periosts eine direkte Beurteilung des Frakturverlaufs schwierig. Deshalb wurden die Kadaverpräparate nach jeder Aufspreizetappe im Osteotomiespalt temporär fixiert und mittels Computertomographie (CT) mit einer Schichtdicke von $1 \mathrm{~mm}$ in 3 Ebenen untersucht. Die statistische Auswertung erfolgte mittels U-Test.

\section{Frakturkriterien}

In den Gruppen der Modellknochen (Gruppe 1-2) wurde eine sichtbare Fissur mit oder ohne Spaltbildung, die vom Biegungsscharnier der lateralen Kortikalis ausging, als Fraktur der Gegenkortikalis gewertet. Analog hierzu
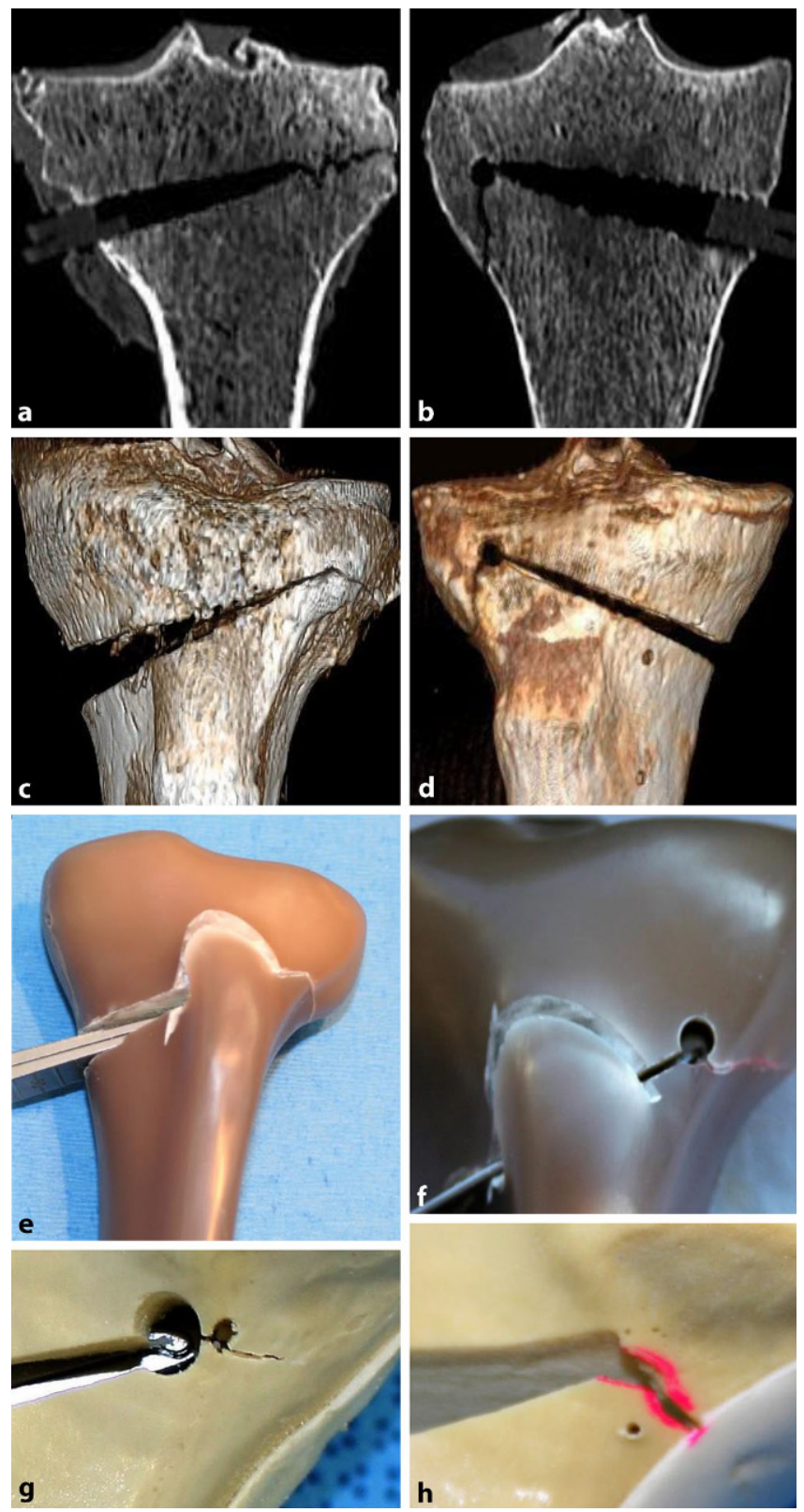

Abb. $4 \Delta$ Beispiele für Frakturtypen Typ 1 und 2 dieser Studie nach der Takeuchi-Klassifikation mit und ohne Entlastungsbohrung. Kadaver: 2-D-CT-Rekonstruktion Typ 1 (a) und Typ 2 (b). 3-D-CT-Rekonstruktion Typ 1 (c) und Typ 2 (d). Sawbone nur Typ 1 (e, f). Synbone beginnender Typ 1 (g) und Typ 2 (h)

wurde bei den Kadaverknochen eine in der CT sichtbare Fissur oder Fraktur der lateralen Kortikalis gewertet.

Die dokumentierten lateralen Kortikalisfrakturen wurden entsprechend der von Takeuchi et al. [1] vorgeschlagenen Klassifikation der lateralen Scharnierfrakturen in 3 Typen eingeteilt:

- Typ 1 stellt eine Verlängerung der horizontalen Osteotomielinie in die laterale Kortikalis dar. Die Fraktur endet unmittelbar oberhalb oder innerhalb des proximalen tibiofibularen Gelenks. Es ist die mit Abstand am häufigsten beobachtete Fraktur. Sie geht i. d. R. mit einem komplikationsarmen Verlauf einher und wird als stabil angesehen.

- Typ 2 zieht in den distalen Teil des proximalen Tibiofibulargelenks. Dieser Frakturtyp ist mit einem protra- 
Tab. 1 Zusammenfassung der Verteilung des Frakturtyps nach Takeuchifür alle Gruppen:86,6\% Typ 1,13,3\% Typ 2. Typ 3 kam nicht vor

\begin{tabular}{|llllllll} 
& $\begin{array}{l}\text { Gruppe 1 } \\
\text { Synbone } \\
\text { ohne Boh- } \\
\text { rung }\end{array}$ & $\begin{array}{l}\text { Gruppe 1 } \\
\text { Synbone } \\
\text { mit Boh- } \\
\text { rung }\end{array}$ & $\begin{array}{l}\text { Gruppe 2 } \\
\text { Sawbone } \\
\text { ohne Boh- } \\
\text { rung }\end{array}$ & $\begin{array}{l}\text { Gruppe 2 } \\
\text { Sawbone } \\
\text { mit Boh- } \\
\text { rung }\end{array}$ & $\begin{array}{l}\text { Gruppe 3 } \\
\text { Kadaver } \\
\text { ohne Boh- } \\
\text { rung }\end{array}$ & $\begin{array}{l}\text { Gruppe 3 } \\
\text { Kadaver } \\
\text { mit Boh- } \\
\text { rung }\end{array}$ \\
\hline Typ 1 & 8 & 8 & 10 & 10 & 7 & 9 \\
Typ 2 & 2 & 2 & 0 & 0 & 3 & 1 \\
Typ 3 & 0 & 0 & 0 & 0 & 0 & 0 \\
\hline
\end{tabular}

hierten Heilungsverlauf verbunden und gilt als instabil [9].

- Typ 3, die laterale Tibiaplateaufraktur, ist die seltenste Variante. Es handelt sich um eine intraartikuläre, stabile Fraktur, die eine präarthrotische Deformität darstellen kann.

Diese Einteilung hat sich in der Klinik als sinnvoll erwiesen, da mit ihr die Stabilität der Knochen-Implantat-Konstruktion und die Notwendigkeit einer Revisionsoperation abgeschätzt werden können.

\section{Ergebnisse}

\section{Synbone-Knochen}

Die Synbone-Modelle (Gruppe 1) konnten sowohl mit als auch ohne Bohrung am weitesten aufgeklappt werden $\left(3^{\circ}\right.$ ohne bzw. 5, $5^{\circ}$ mit Bohrung). Die entlastende Bohrung der Gegenkortikalis führte im Durchschnitt zu einer Vergrößerung des Aufklappwinkels bis zur Frakturentstehung von $2,5^{\circ}$ (ca. $83 \% ; p=0,001$ ).

\section{Sawbone-Knochen}

Bei der Standardosteotomie sind bei $3^{\circ}$ Aufspreizung bereits alle Modelle gebrochen. Der durchschnittliche Winkel lag bei $2,2^{\circ}$ ohne Bohrung und konnte mit Entlastungsbohrung um $109 \%$ auf durchschnittliche $4,6^{\circ}$ gesteigert werden, bis auch hier die Gegenkortikalis brach. Der Unterschied erwies sich als statistisch signifikant $(p<0,001)$.

\section{Kadaverknochen}

Die Kadaverknochen konnten ohne entlastende Bohrung der Gegenkortikalis bis durchschnittlich $2,9^{\circ}$ ohne das Auftre- ten einer Fraktur aufgeklappt werden. Die Bohrung führte hier zu einer Vergrößerung des maximalen Winkels um ca. $44 \%$, aufdurchschnittliche $4,2^{\circ}$. Beizu großer Streubreite der Ergebnisse waren die Unterschiede hier nicht signifikant $(p>0,1)$.

\section{Verteilung des Frakturtyps nach Takeuchi}

Insgesamt 52 der 60 Frakturen im Biegungsscharnier waren gemäß der Takeuchi-Klassifikation [1] dem Typ 1 zuzuordnen und demnach als stabil anzusehen. Die übrigen 8 Frakturen im Biegungsscharnier gehörten zum instabilen Frakturtyp 2 mit nach lateral absteigendem Verlauf in den distalen Anteil des proximalen tibiofibularen Gelenks, wovon sich 5 in der Gruppe der Osteotomien ohne Entlastungsbohrung fanden. Der Frakturtyp 3 fand sich in keiner der untersuchten Präparate dieser Studie (• Tab. 1; - Abb. 4a-h).

\section{Zusammenfassung der Ergebnisse}

Der durchschnittliche maximale Winkel lag ohne Entlastungsbohrung für alle 3 Gruppen zusammen bei $2,7^{\circ}$ und mit Bohrung bei $4,76^{\circ}$, was einem Zuwachs von $2,06^{\circ}(+76,5 \%)$ entspricht (• Abb. 1). In jeder Gruppe führte die Entlastungsbohrung zu einer Vergrößerung des Aufklappwinkels, bevor es zu einer Fraktur kam. Prozentual war dieser Effekt bei den Sawbone-Modellen am deutlichsten. Hier vergrößerte sich der Winkel statistisch signifikant $(p<0,001)$ um $2,4^{\circ}$ von $2,2^{\circ}$ auf $4,6^{\circ}(+104 \%)$. In der SynboneGruppe betrug der ebenfalls signifikante Zuwachs $(p<0,005) 2,5^{\circ}(+83 \%)$, und bei den Kadaverknochen kam es zu einem Zuwachs von $1,3^{\circ}(+44 \%)$; jedoch zeig- te sich hier keine statistische Signifikanz ( $p$ > 0,1; - Abb. 5). 52 der 60 (86,6\%) Frakturen im Biegungsscharnier waren gemäß der Takeuchi-Klassifikation [1] vom Typ 1. Lediglich 8 Frakturen (13,3\%) im Biegungsscharnier gehörten zum instabilen Frakturtyp 2, mit nach lateral absteigendem Verlauf in den distalen Anteil des proximalen tibiofibularen Gelenks. Der Frakturtyp 3 fand sich in keiner der untersuchten Präparate dieser Studie. Ein signifikanter Unterschied bezüglich der Entlastungsbohrung bestand nicht.

\section{Diskussion}

In dieser Studie wurde untersucht, ob eine entlastende anteroposteriore Bohrung durch das Biegungsscharnier („hinge“) am lateralen Ende der horizontalen Osteotomie bei der aufklappenden hohen Tibiaosteotomie die Fraktur der Gegenkortikalis verhindert. Hierzu wurden zwei verschiedene Kunstknochenarten sowie Kadaverknochen in 3 Untersuchungsgruppen eingeteilt. Alle Tibiae wurden einer aufklappenden Osteotomie in Standardtechnik unterzogen. Die Hälfte aller Tibiae pro Gruppe wurde zusätzlich mit einer Entlastungsbohrung im Biegungsscharnier versorgt. Beim schrittweisen Aufklappen wurde die Integrität der lateralen Kortikalis im Biegungsscharnier dokumentiert.

Ein wichtiges Ergebnis dieser Untersuchung ist, dass bei den Tibiae aller Untersuchungsgruppen durch die Bohrung im Biegungsscharnier, der Winkel der aufklappenden Osteotomie bis zur Fraktur der Gegenkortikalis um maximal 2,06 ${ }^{\circ}$ vergrößert werden konnte. Statistisch signifikant war der Effekt nur bei den beiden Kunstknochen. Offensichtlich wiesen die humanen Präparate eine hohe interindividuelle Variabilität der Knochenqualität auf, so dass der Unterschied zwischen der Gruppe mit und ohne Bohrung nicht signifikant war.

Zweitens ist festzustellen, dass es auch mit einer Entlastungsbohrung schon bei Korrekturwinkeln um $5^{\circ} \mathrm{zu}$ einer Fraktur der Gegenkortikalis kam. Zwar scheint der prozentuale Steigerungseffekt des frakturfreien Aufspreizens im Osteotomiespalt durch die Bohrung im Biegungsscharnier hoch $\mathrm{zu}$ sein, der 


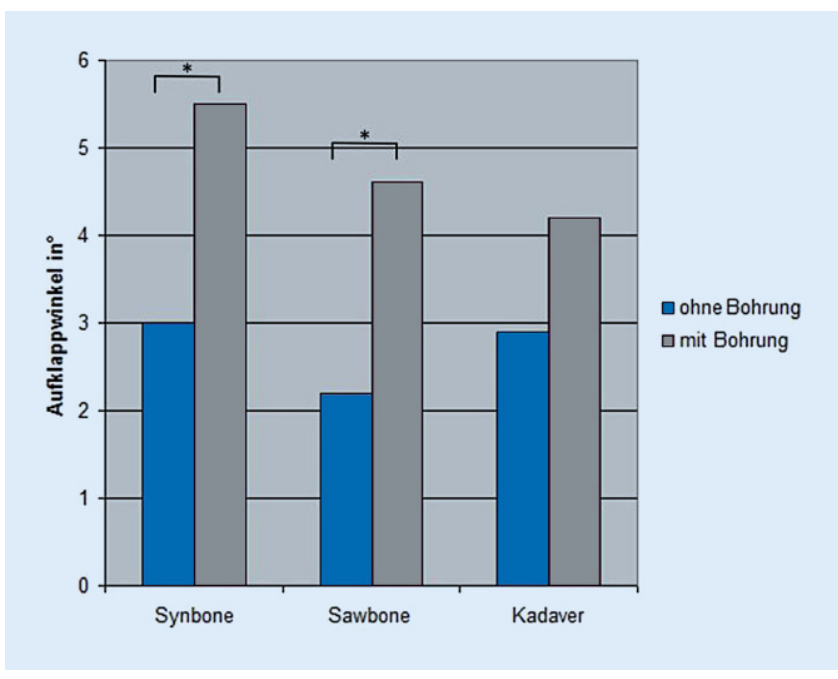

Abb. $5 \Delta$ Vergleich der Aufspreizwinkel mit und ohne Entlastungsbohrung: Bei einem Korrekturwinkel von ca. $5^{\circ}$ (Synbone, Gruppe 1, und Sawbone, Gruppe 2) wird trotz der anteroposterioren Bohrungen im lateralen Biegungsscharnier die Kapazität zur elastischen Deformierung überschritten, so dass eine plastischen Verformung mit Fraktur der Gegenkortikalis auftreten kann $\left({ }^{*} p<0,005\right)$

absolute Zuwachs beträgt aber nur etwas mehr als $2^{\circ}$. Daher scheint diese Bohrung im Biegungsscharnier unter den beschriebenen Versuchsbedingungen nur bei Osteotomien mit einem maximalen Korrekturbedarf von $5^{\circ}$ eine klinische Bedeutung zu haben.

In Bezug auf den Frakturtyp nach Takeuchi, überwog der mit den geringsten Komplikationen verbundene Typ I bei den Tibiae aller Untersuchungsgruppen (86,7\%; [9]). Frakturtyp 2 fand sich insgesamt nur 8-mal. Es konnte kein signifikanter Unterschied zwischen einer klassischen, linear endenden Osteotomie und einer Osteotomie mit Bohrung im Scharnier bezüglich der Verteilung des Frakturtyps festgestellt werden. Auch trat bei keiner der insgesamt 60 Osteotomien eine intraartikuläre (Тyp 3) Fraktur nach Takeuchi [1] auf.

Die homogene Verteilung der Frakturtypen innerhalb und zwischen den Untersuchungsgruppen kann an der Nähe des Endpunkts der horizontalen Osteotomie liegen. Dieser Endpunkt war nur ca. $8 \mathrm{~mm}$ entfernt von der lateralen Kortikalis, so dass die Entwicklung einer Typ-1-Fraktur dem Weg der geringsten Risswachstumslänge entsprechen könnte und lediglich die Verlängerung der horizontalen Osteotomielinie in die laterale Kortikalis darstellte (• Abb. 6a, b).
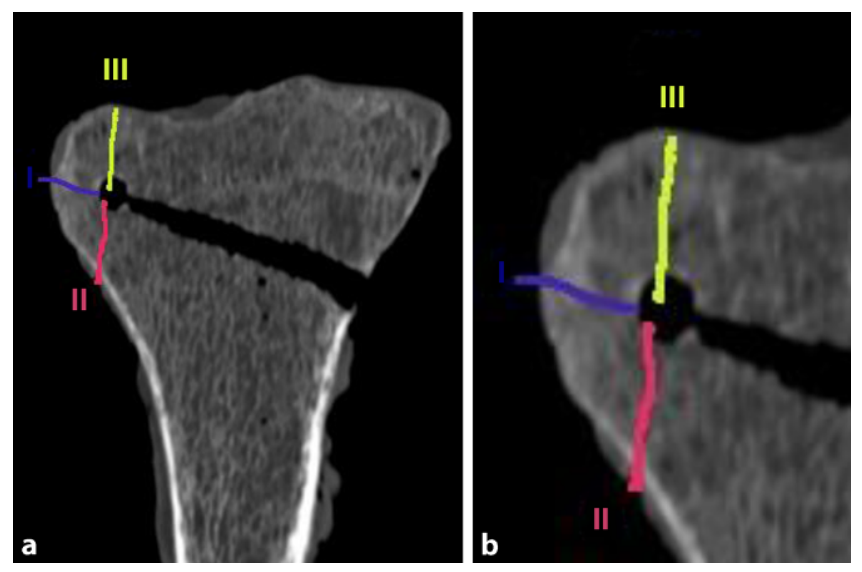

Abb. 6 \& Schematisches Anzeichnen (a) und Vergrößerung (b) der 3 Frakturtypen nach Takeuchi [1]. Blau stabiler Typ I, rot instabiler Typ II, gelb intraartikulärer Typ 3

Tendenziell scheinen die Knochen mit einer höheren Materialdichte, passend zur Knochenanatomie des jüngeren Patienten (Untersuchungsgruppe 1 und 2), eher von einer stressreduzierenden und frakturprotektiven Bohrung im Biegungsscharnier zu profitieren als Knochen mit einer geringeren Materialdichte (Untersuchungsgruppe 3).

Allerdings relativiert sich der Zusammenhang zwischen den Materialeigenschaften der Prüfkörper dieser Studie und dem frakturprotektiven Effekt der Bohrung, da dies nur für Korrekturwinkel bis ca. $5^{\circ}$ gilt. Korrekturen, die $5^{\circ}$ überschreiten, münden in dieser Studie ausnahmslos in eine Fraktur der Gegenkortikalis, unabhängig von der Existenz einer Bohrung im Biegungsscharnier.

\section{Limitationen des Studiendesigns}

Auch in klinischen Studien wird die Fraktur der Gegenkortikalis regelmäßig beschrieben. Es fällt jedoch auf, dass auch bei Korrekturen über $8^{\circ}$ nicht alle Patienten eine Fraktur der Gegenkortikalis aufweisen $[6,13]$. Dies steht im deutlichen Gegensatz zu den Labordaten dieser Studie. Eine mögliche Erklärung für die höhere Inzidenz der Fraktur der Gegenkortikalis in der vorliegenden Studie ist die Tatsache, dass sowohl die verwendeten Knochenmodelle als auch die humanen Kadaver weder einen Kapsel-Band-Apparat noch eine muskuläre Stabilisierung aufwiesen. Die Beurteilung der Integrität der Gegenkortikalis erfolgte also in die- 
ser Studie nach dem Alles-oder-NichtsPrinzip. In der klinischen Praxis hingegen werden Fissuren und Frakturen im Biegungsscharnier durch die Ligamentotaxis der Weichteile (Kapsel, Sehnen und Bänder) stabilisiert. Darüber hinaus können kleine Fissuren intraoperativ nicht mit einem Bildwandler dargestellt werden, während in der vorliegenden Studie CT-Schnitte mit einem Abstand von $1 \mathrm{~mm}$ durchgeführt wurden, um diese Fissuren dokumentieren zu können.

\section{Fazit für die Praxis}

- Bei den Kunstknochen aus Verbundmaterial (Sawbone und Synbone) führte die anteroposteriore Bohrung der Gegenkortikalis des horizontalen Osteotomieschenkels einer biplanaren Osteotomie des Tibiakopfes zu einer signifikanten Vergrößerung des Aufspreizwinkels um durchschnittlich $2,1^{\circ}$ auf maximal $4,8^{\circ}$, bis zum Auftreten der Fraktur.

- Dieser frakturverhindernde Effekt im Biegungsscharnier war bei den osteoporotischen Kadaverknochen nicht signifikant ausgeprägt.

- Unabhängig von der Konsistenz der Tibiae und dem Vorhandensein einer AP-Bohrung zeigte sich bei allen Präparaten eine Fraktur im Biegungsscharnier ab einem Korrekturwinkel von $>5^{\circ}$.

- Ein frakturprotektiver Effekt durch das anteroposteriore Aufbohren des Biegungsscharniers zeigte sich in dieser Studie nur bei kleineren Korrekturen, wie sie im klinischen Alltag, z. B. zur Entlastung von knorpelsanierenden Eingriffen (OATS, ACT, Mikrofrakturierung), ohne begleitende Fehlstellung durchgeführt werden.

- Für die Korrektur einer Varusfehlstellung, bei der regelmäßig Korrekturwinkel $>8^{\circ}$ notwendig sind, scheint der frakturverhindernde Effekt einer anteroposterioren Bohrung im Biegungsscharnier unter den Testbedingungen der vorliegenden Studie keine Bedeutung zu haben.

\section{Korrespondenzadresse}

Prof. Dr. D. Pape

Orthopädische Klinik des Centre Hospitalier de Luxembourg, Akademisches Lehrkrankenhaus der Universitätskliniken des Saarlandes 78, rue d'Eich, 1460 Luxembourg, Luxemburg dietrichpape@yahoo.de

\section{Einhaltung ethischer Richtlinien}

Interessenkonflikt. G. Reyle, O. Lorbach, A. Diffo Kaze, A. Hoffmann und D. Pape geben an, dass kein Interessenkonflikt besteht.

Dieser Beitrag beinhaltet keine von den Autoren durchgeführten Studien an Menschen oder Tieren.

Open Access. Dieser Artikel wird unter der Creative Commons Namensnennung 4.0 International Lizenz (http://creativecommons.org/licenses/by/4.0/deed. de) veröffentlicht, welche die Nutzung, Vervielfältigung, Bearbeitung, Verbreitung und Wiedergabe in jeglichem Medium und Format erlaubt, sofern Sie den/die ursprünglichen Autor(en) und die Quelle ordnungsgemäßnennen, einen Link zur Creative Commons Lizenz beifügen und angeben, ob Änderungen vorgenommen wurden.

\section{Literatur}

1. Takeuchi $R$, Ishikawa $H$, Kumagai $K$, Yamaguchi Y, Chiba N, Akamatsu Y, Saito T (2012) Fractures around the lateral cortical hinge after a medial opening-wedge high tibial osteotomy: a new classification of lateral hinge fracture. Arthroscopy 28:85-94

2. Cole BJ, Harner CD (1999) Degenerative arthritis of the knee in active patients: evaluation and management. J Am Acad Orthop Surg 7:389-402

3. Lobenhoffer P, Agneskirchner JD (2003) Improvements in surgical technique of valgus high tibial osteotomy. Knee Surg Sports Traumatol Arthrosc 11:132-138

4. Lobenhoffer P, Agneskirchner J, Zoch W (2004) Open valgus alignment osteotomy of the proximal tibia with fixation by medial plate fixator. Orthopäde 33:153-160

5. Staubli AE, De Simoni C, Babst R, Lobenhoffer P (2003) TomoFix: a new LCP-concept for open wedge osteotomy of the medial proximal tibia early results in 92 cases. Injury34(Suppl2):B55-B62

6. Bae DK, Park CH, Kim EJ, Song SJ (2016) Medial cortical fractures in computer-assisted closingwedge high tibial osteotomy. Knee 23:295-299

7. Kessler OC, Jacob HA, Romero J (2002) Avoidance of medial cortical fracture in high tibial osteotomy: improved technique. Clin Orthop Relat Res 395:180-185

8. Pape D, Adam F, Rupp S, Seil R, Kohn D (2004) Stability, bone healing and loss of correction after valgus realignment of the tibial head. A roentgen stereometry analysis. Orthopäde 33:208-217

9. Schroter S, Ateschrang A, Ihle C, Stockle U, Konstantinidis L, Dobele S (2014) Lateral hinge fractures in open wedge high tibial osteotomy. Orthopäde 43:1000-1007
10. van Houten $A H$, Heesterbeek PJ, van Heerwaarden $\mathrm{RJ}$, van Tienen TG, Wymenga AB (2014) Medial open wedge high tibial osteotomy: can delayed or nonunion be predicted? Clin Orthop Relat Res 472:1217-1223

11. van Raaij TM, Brouwer RW, de Vlieger R, Reijman $M$, Verhaar JA (2008) Opposite cortical fracture in high tibial osteotomy: lateral closing compared to the medial opening-wedge technique. Acta Orthop 79:508-514

12. Han SB, Lee DH, Shetty GM, Chae DJ, Song JG, Nha KW (2013) A "safe zone" in medial open-wedge high tibia osteotomy to prevent lateral cortex fracture. Knee Surg Sports Traumatol Arthrosc 21:90-95

13. Woodacre T, Ricketts M, Evans JT, Pavlou G, Schranz P, Hockings M (2016) Complications associated with opening wedge high tibial osteotomy a review of the literature and of 15 years of experience. Knee 23:282

14. Diffo KA, Maas S, Waldmann D, Zilian A, Dueck K, Pape D (2015) Biomechanical properties of five different currently used implants for open-wedge high tibial osteotomy. J Exp Orthop 2:14

15. Maas S, Diffo KA, Dueck K, Pape D (2013) Static and dynamic differences in fixation stability between a spacer plate and a small stature plate fixator used for high tibial osteotomies: a biomechanical bone composite study. ISRN Orthop 2013:387620. doi:10.1155/2013/387620

16. Pape D, Kohn D, van Giffen N, Hoffmann A, Seil R, Lorbach $O$ (2013) Differences in fixation stability between spacer plate and plate fixator following high tibial osteotomy. Knee Surg Sports Traumatol Arthrosc 21:82-89

17. Chong AC, Miller F, Buxton M, Friis E (2007) Fracture toughness and fatigue crack propagation rate of short fiber reinforced epoxy composites for analogue cortical bone. J Biomech Eng 129:487-493

18. Gardner MP, Chong ACM, Pollock AG, Wooley PH (2010) Mechanical evaluation of large-size fourthgeneration composite femur and tibia models. Ann Biomed Eng 38:613-620

19. Heiner A (2008) Structural properties of fourthgeneration composite femurs and tibias.JBiomech 41:3282-3284

20. Staubli AE, Jacob HA (2010) Evolution of openwedge high-tibial osteotomy: experience with a special angular stable device for internal fixation without interposition material. Int Orthop 34:167-172

21. Fowler P, Tan L, Brown G (2000) Medial opening wedge high tibial osteotomy: How I do it. Oper Tech Sports Med 8:32-38

22. Pape D, Seil R, Adam F, Rupp S, Kohn D, Lobenhoffer P (2004) Imaging and preoperative planning of osteotomy of tibial head osteotomy. Orthopäde 33:122-134 